\title{
Téoros
}

Revue de recherche en tourisme

\section{Thomas Cook en Égypte et à Louxor}

L'invention du tourisme moderne au XIX ${ }^{\mathrm{e}}$ siècle

\section{Sandrine Gamblin}

Volume 25, numéro 2, été 2006

Désirs d’Orient : du passé vers l'avenir

URI : https://id.erudit.org/iderudit/1071063ar

DOI : https://doi.org/10.7202/1071063ar

Aller au sommaire du numéro

Éditeur(s)

Université du Québec à Montréal

ISSN

0712-8657 (imprimé)

1923-2705 (numérique)

Découvrir la revue

\section{Citer cet article}

Gamblin, S. (2006). Thomas Cook en Égypte et à Louxor : l'invention du tourisme moderne au XIX ${ }^{\mathrm{e}}$ siècle. Téoros, 25(2), 19-25.

https://doi.org/10.7202/1071063ar d'utilisation que vous pouvez consulter en ligne.

https://apropos.erudit.org/fr/usagers/politique-dutilisation/ 


\section{Thomas Cook en Égypte et à Louxor L'invention du tourisme moderne au XIXe siècle}

\section{Sandrine Gamblin}

En 1869, l'ouverture du canal de Suez est célébrée en présence des représentants des puissances coloniales et de l'aristocratie européennes. À cette occasion, Thomas Cook fait affréter deux bateaux de croisière selon la formule combinée du voyage en groupe et du traveler check. Le premier permet de réduire les coûts et de présenter l'offre à un public élargi. Le second libère le voyageur de tous soucis matériels et facilite l'organisation pratique. Dès sa première mise en œuvre en 1868 en Suisse, la formule fait des émules, favorisée en outre par la révolution des transports que connaît le XIXe siècle, et est appliquée pour la première fois hors des frontières européennes en 1869, à l'occasion des festivités égyptiennes. La croisière sur le Nil ne relève plus alors du seul privilège de l'aristocratie. Au grand désespoir des voyageurs contemplatifs, les "hordes de Cooks et Cookesses" (Loti, 1909) envahissent progressivement les temples à mesure que les bourgeoisies européennes accèdent à la pratique des loisirs et que l'égyptologie suscite l'intérêt du grand public.

La diffusion des connaissances sur l'Orient contribue notamment à la diversification des candidats au voyage (Berchet, 1985 ; Rauch, 2000). Elle passe notamment par la publication d'ouvrages d'un genre nouveau où l'on trouve à la fois de l'information historique et culturelle et des conseils pratiques. L'invention du guide touristique est un élément déterminant des transformations pratiques du voyage, à la fois portée par une demande touristique croissante et un souci de vulgariser des savoirs jusque-là réservés aux initiés. Jusque dans les années 1870, le guide touristique sur l'Égypte reste d'un genre littéraire et élitiste, à l'instar du Riffaud, paru en 1830. « L'apparition d'un étranger est

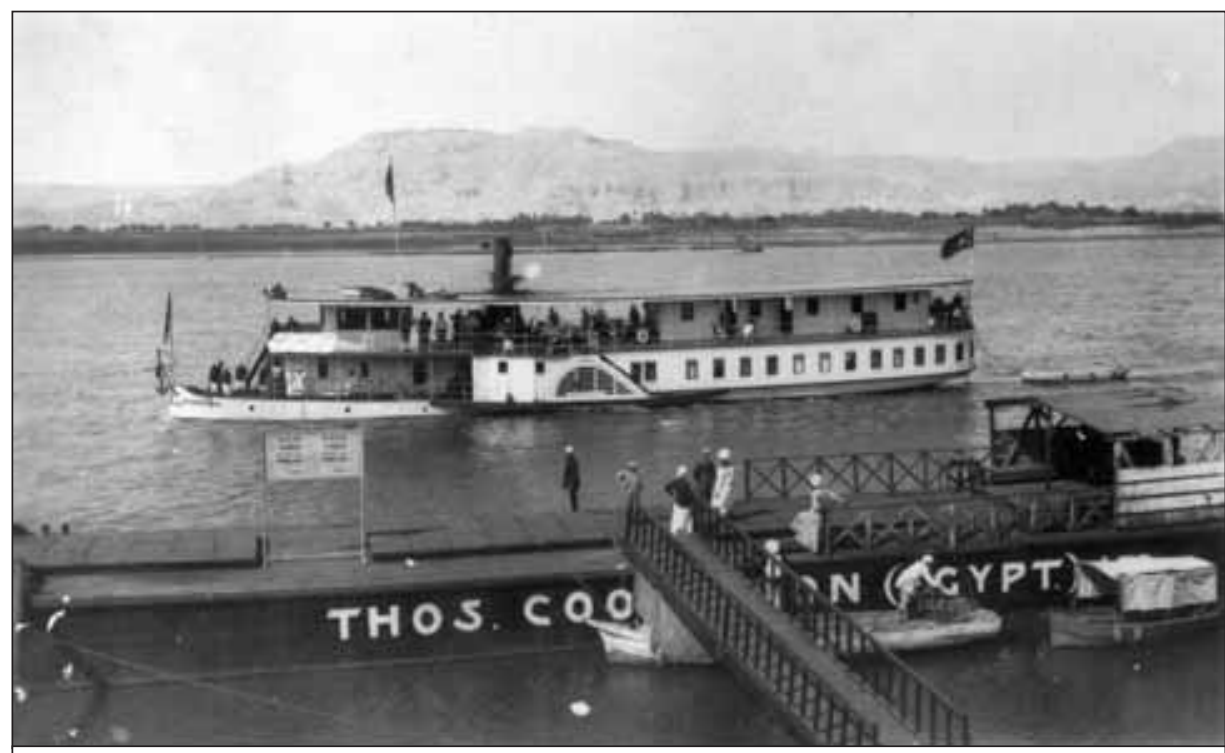

Embarcadère de l'agence Thomas Cook \& Son à Louxor (Égypte).

Photo : Carte postale Gaddis, non-datée ${ }^{1}$.

un accident qui étonne » alors encore (Volkoff, 1967: 23). Le terme de touriste n'est d'ailleurs pas encore passé dans la langue française (Urbain, 1991). La « démocratisation » progressive du voyage en Orient encourage les éditeurs à s'intéresser sous un angle plus commercial à l'Égypte. Les éditions Baedecker y consacrent un premier ouvrage en 1877 (1878 pour la version anglaise) et connaît une demi-douzaine de rééditions en vingt ans: "L'Égypte est, comme aucun autre pays de l'Orient ou même du monde, le pays classique de l'Histoire, le berceau de la civilisation sur lequel viennent à notre rencontre à chaque instant les monuments anciens " (cité par Volkoff, 1967 : 27). Le guide se veut surtout pratique: conseils vestimentaires (pour ou contre la tenue locale), suggestions de circuits, information égyptologique simplifiée, présentation des mœurs locales et mise en garde sur la rencontre avec l'autochtone. De 1890 à 1914, près de 80 ouvrages du genre sont édités sur l'Égypte, principalement en anglais et en français. C'est dire l'importance que pouvait alors constituer ce pays aux yeux des professionnels du voyage et dans les esprits en général (Reid, 2002 : 299).

Outre la littérature et les ouvrages consacrés au voyage en Orient, les événements publics de type exposition universelle contribuent à façonner les imaginaires des candidats au voyage durant la seconde moitié du XIXe siècle. La première participation de l'Égypte en 1867 à l'exposition universelle de Paris fait connaître au monde occidental les trésors d'une civilisation ancienne aux fondements de l'histoire de I'humanité. Dès lors, l'Égypte participe régulièrement aux principaux événements du genre, de Vienne à Lyon et jusqu'à Philadelphie (Dyab, 1994: 60). Éditions touristiques, expositions universelles, vulgarisation des savoirs égyptologiques et égyptomanie occidentale qui en résultent 
(Humbert et al., 1994), concourent tous à faire de l'Égypte une destination privilégiée du tourisme occidental en pleine émergence. L'agence Thomas Cook est à ce titre, à partir de 1869, un acteur majeur de la mise en tourisme de l'Égypte. Est-ce une simple occurrence de dates, entre le premier voyage organisé par Thomas Cook et l'inauguration du canal de Suez ? Le développement de l'agence dans les deux décennies qui suivent démontre que son rôle et ses ambitions dépassent le simple secteur touristique et sont étroitement liés aux intérêts de l'empire britannique dans le pays.

\section{Thomas Cook en Égypte : de la croisière sur le Nil aux campagnes militaires}

Le 17 novembre 1869, Thomas Cook est invité aux festivités d'inauguration du canal de Suez. Pour l'événement, deux bateaux de touristes partent rejoindre la flotte roya$1 e^{2}$. Miss Riggs, dame anglaise embarquée dans cette première équipée de roturiers à qui la formule est destinée, livre ses émotions et donne le ton dans ses carnets de voyage $^{3}$. La liste des passagers est ouverte aux classes bourgeoises, aux employés de banques, aux commerçants ou à la profession libérale et le décalage des standings nourrit les sarcasmes de la presse britannique, dont les caricatures ridiculisent les Cooks et Cookesses à la recherche éperdue des divas de l'aristocratie cosmopolite. II s'agit d'être vu et surtout de voir les Grands de ce monde conviés aux festivités, même à distance respectable. Cependant, la déception est grande pour Miss Riggs : Seule la dahabiya de Lady Duff Gordon est entrevue à Edfû : "[She] was in her dahabieh near but we did not see her. Lady Gordon is in decline-the reason of her exile-more particularly interested in the fellaheen. " De plus, la croisière est semée d'obstacles administratifs, notamment à l'arrivée à Louxor. Le droit de passage des bateaux de Mr. Cook semble contesté par les autorités locales, comme l'observe notre touriste: "Mister Cook would not put pen to paper as he had so little power over the Viceroy's regulations. " Arrivés trop tard dans l'antique capitale thébaine, Miss Riggs et le groupe de touristes ne peuvent assister à l'illumination du temple de Karnak. Au-delà des anecdotes rapportées, les notes de voyage de Miss Riggs, figure « veblennienne » par excellence (Veblen, 1899), évoquent plus largement les transformations des sociétés industrielles durant la seconde moitié du XIXe siècle et l'émergence d'une classe des loisirs et de consommation qui constitue de nos jours le marché économique le plus dynamique dans le monde. L'agence Thomas Cook joue un rôle prépondérant dans la genèse du tourisme international, dont l'Égypte constitue une destination stratégique, une sorte de laboratoire de mise en place des conditions de son expansion.

Dès 1870, John Mason, fils de Thomas Cook, reprend la direction de l'agence, avec des ambitions affichées de développer les activités de celle-ci. Le fils Cook est résolument plus téméraire que son père en affaires. La compagnie fait alors l'acquisition de deux bateaux à vapeur destinés à la navigation sur le Nil et obtient en 1875 un contrat d'exclusivité pour l'acheminement du courrier postal vers l'Égypte. Les autorités égyptiennes lui concèdent dans un premier temps le droit de circuler entre Assouan et la première cataracte. Rapidement, la plupart des métiers liés au tourisme et au transport sont placés sous le contrôle de l'agence, non sans susciter les protestations de certains professionnels. En 1874, les drogmans égyptiens, ancêtres du guide touristique, protestent dans le London Times contre l'intrusion des "agents anglais " recrutés par Thomas Cook $^{4}$. Le monopole de Thomas Cook en Égypte suscite les commentaires de nombreux observateurs, tel Stevens, correspondant pour le Daily Mirror en 1898 :

The nominal suzerain of Egypt is the Sultan; its real suzerain is Lord Cromer. Its nominal Governor is the Khedive; its real governor, for a final touch of comic opera, is Thomas Cook \& Son [...]. Mr. Cook is a blessing to Egypt. Perhaps the only one of Egypt's recent blessings which nobody disputes. It is not only the vast amount of money he brings into the country, nor the vast number of people he directly employs. Besides that you will find natives all up the Nile who practically live on him. Those donkeys are subsidised by Cook: that little plot of lettuce is being grown for Cook, and so are the fowls; these boats tied up on the bank were built by the sheikh of the Cataracts for the tourist service with money advanced by Cook (Stevens, 1898: 60 et 271-272).
En effet, Thomas Cook \& Son, qui opérait déjà en maître dans les secteurs du tourisme et des transports, a aussi étendu ses activités aux affaires militaires. La crise politique qui secoue le pays dans les années 18811884 (révolte conduite par Urâbî, imposition du mandat britannique, expédition soudanaise) met un frein aux arrivées touristiques vers l'Égypte. À la demande des autorités britanniques, l'agence organise les renforts pour venir en aide à l'expédition de Gordon, en déroute au Soudan, et fait embarquer sur ses bateaux jusqu'à 18000 hommes, 40000 tonnes de vivres et d'armements et autant de charbon. Pas moins de 28 bateaux à vapeurs et 650 bateaux à voile opèrent sur le Nil, acheminent troupes, armements et vivres, mobilisant pour la logistique plus de 5000 hommes. Malgré un tel déploiement, le général Gordon tombe à Khartoum en janvier 1885. La défaite est cuisante pour la Couronne britannique. En revanche, Thomas Cook \& Son consolide ses positions dans les affaires de l'empire et de l'Égypte. Pour d'autres, l'engagement au Soudan semble avoir aussi porté quelques compensations à la défaite, notamment dans le secteur touristique. Ainsi, Georges Nungovich, qui avait commencé sa carrière en Égypte comme portier à la gare de Ramsès, s'engage aux côtés des troupes britanniques au Soudan. À son retour, il acquiert la résidence khédiviale située au pied des pyramides de Guiza et la convertit en un hôtel de luxe - la Mena House, établissement désormais mythique (Nelson, $1997: 25)$.

L'historiographie égyptienne ne fait guère mention du rôle de Thomas Cook dans la campagne du Soudan; ni ne relate d'ailleurs le rôle de la compagnie dans les intérêts de l'empire britannique en Égypte en général. L'émergence du tourisme dans ce pays, ainsi que plus généralement le rôle de Thomas Cook dans I'histoire du tourisme international, s'inscrivent pourtant indéniablement dans le sillon des projets coloniaux au XIXe siècle :

[...] it must be said that the connection between tourism and empire, where Egypt was concerned, was much more than a matter of ironic comparison: for it was in Egypt that an institution of the tourist industry first went to work in direct and indirect collaboration with British imperial interest (Brendon, 1991 : 323). 
Inscrite comme l'étape incontournable du voyage en Orient et de la croisière sur le Nil, la bourgade de Louxor, ancienne capitale de Thèbes, doit sa raison d'être et son développement urbain au tourisme et à l'exploitation de ses richesses archéologiques. Thomas Cook, par son rôle en Égypte à la fin du XIXe siècle, y joue un rôle de premier plan.

\section{Du village à la ville cosmopolite : fabrique patrimoniale et touristification de Louxor}

Lorsque Miss Riggs arrive à Louxor en 1869, elle ne voit guère plus que des ruines antiques et un village sans grande activité. Parmi les 14 colonnes qui émergent du sable et constituent la cour centrale du temple de Louxor, les habitations du village dit « arabe " se sont construites autour de la mosquée du saint Abû Hajjâj, patron de la ville (Chih, 1993). Cette mosquée est construite sur les fondations d'une église et accueille tous les ans un pèlerinage dont les caractéristiques ne vont pas sans rappeler certains rites pharaoniques (Legrain, 1914). Le temple et ses occupants synthétisent en plusieurs siècles d'histoire du peuplement de la région des périodes pharaonique et copte à la conquête musulmane. La famille des Hajjâîi, dignes descendants du saint patron, administre les lieux de culte et de pèlerinage et règne sur les activités du bazar, ouvert durant la haute saison touristique, de décembre à février. Louxor accueille en outre une communauté copte importante ${ }^{5}$, au sein de laquelle quelques familles règnent sur les terres agricoles et les représentations consulaires, à l'instar des Andrawos, consuls de père en fils et antiquaires célèbres, dont les villas construites au début du siècle dernier jouxtent encore aujourd'hui le site antique sur les bords du $\mathrm{Nil}^{6}$.

Dès 1876, la croisière sur le Nil constitue un produit-phare de l'agence Cook:

The number of passengers for the Nile Steamers have been greater than could be comfortably accommodated, and the only complaints we have received arise from overcrowding. To remedy this for next season, we have arranged for a weekly service of the Nile Steamers during the months of January, February and March (The Excursionist ${ }^{7}$ ).

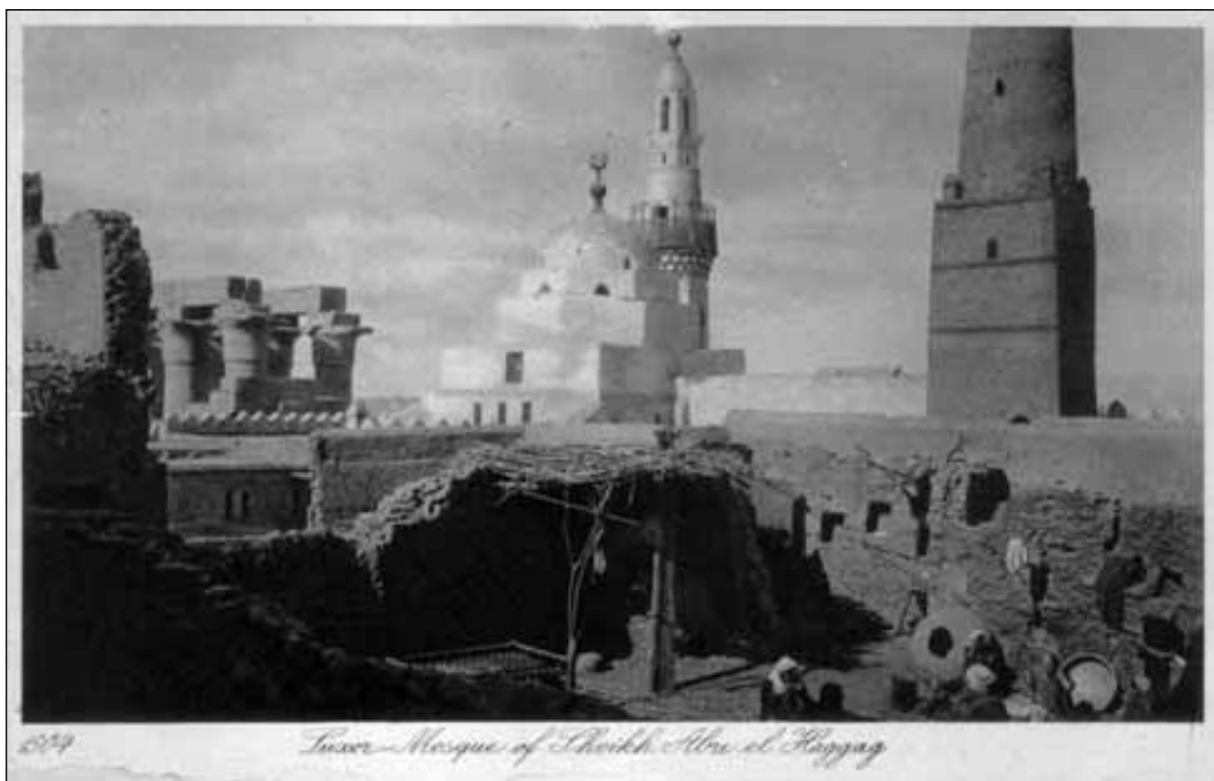

Carte postale fin XIXè. Louxor (Égypte), le village au premier plan, le temple et la mosquée au second. Photo : Collection personnelle de l'auteure

L'investissement de l'agence dans l'amélioration des bateaux et des services fait de la croisière sur le Nil un produit tout à fait recommandable à partir de 1880. John Mason Cook, alors propriétaire de neuf bateaux à vapeur, obtient l'exclusivité de la gestion du trafic fluvial et de sa main-d'œuvre pour une période de dix ans. II en conserve le contrôle jusqu'au début du XXe siècle, avec une flotte qui atteint en 1902 jusqu'à 22 unités (Brendon, 1991).

Lorsque Loti revient à Louxor durant I'hiver 1907, il ne peut que constater avec amertume les transformations récentes de la bourgade.

C'est fini de Louxor! Et quelle affluence de monde, ici ! [...] Tout au long des berges, il y a une rangée de ces bateaux touristes, espèces de casernes à deux ou trois étages, qui de nos jours infestent le Nil depuis le Caire jusqu'aux cataractes - et ils sifflent, et leurs dynamos font un intolérable vacarme trépidant [...] (Loti, 1909).

Les touristes étrangers, Cooks and Cookesses, sont «quelques milliers » chaque année à Louxor et en Haute-Égypte en 1914, selon G. Legrain (Legrain, 1914). La ville vit en grande partie au rythme de ses occupants étrangers, touristes, voyageurs, savants et administrateurs, à savoir du mois d'octobre au mois d'avril, la haute saison se situant de janvier à mars. Il est difficile d'évaluer leur importance. Selon Maspero, l'Égypte compte en 1901 « deux mille touristes au moins» (1901: 89). En 1884, lorsque Maspero doit financer son projet de déblaiement du temple de Louxor, il dénombre en Égypte jusqu'à « [...] 3000 voyageurs environ qu'attirent non les factories de sucre, de coton et de chemins de fer, et tout ce que l'on qualifie d'utile et de productif, mais tous les monuments de l'art arabe et égyptien ${ }^{8}$.

Louxor, ancienne capitale thébaine, devient l'étape incontournable de la croisière sur le Nil. En deux décennies, le village construit au cœur du temple de Louxor s'étend hors les murs et devient une ville cosmopolite où se rencontre chaque hiver la bonne société européenne. La transformation est particulièrement radicale et la géographie des lieux est littéralement bouleversée, d'une part par les programmes de fouilles archéologiques, d'autre part par le développement concomitant du tourisme.

À quoi peut donc ressembler une bourgade de Haute-Égypte à la fin du XIXe siècle? Louxor est, en 1880, «a large village, increasing both in population and prosperity» (A Handbook for Travellers, 1880). Pour moitié enfoui dans le sable, le temple de Louxor constitue un lieu surélevé jusqu'atteindre six mètres au-dessus du sol antique et protége ainsi des crues du Nil le 
village dit «arabe». Celui-ci comptait alors "trente maisons et quatre-vingt paillotes [qui] s'appuyaient au fût des colonnes, se collaient le long des murailles et écrasaient les architraves du poids de leurs briques "9. Vers le nord, le dromos bordé de sphinx criocéphales est partiellement visible, découvert au niveau de la place du Marché (Daressy, 1893), et reliant les temples de Louxor et de Karnak. Sur la partie sud du temple, se trouvaient jusque dans les années 1880 les locaux de la police et la Maison de France ${ }^{10}$. Enfin, accolé au pylône ouest du temple, jouxtant l'obélisque restant (son jumeau étant à Paris sur la place de la Concorde), se dresse la maison d'hôtes des Hajjâjî, descendants du saint patron, face à la rue principale de la bourgade qui traverse vers le nord le site antique en direction de Karnak.

Rappelons que l'occupation des sites antiques n'est pas une caractéristique propre à Louxor et se retrouve tout le long de la vallée du Nil. Les villages limitrophes sont situés au cœur même des ruines : le village de Karnak est installé dans le temple sud et s'est étendu sur une grande partie du site antique. À Gurna, sur la rive ouest, le village fut longtemps accolé au temple de Séthi $1^{\mathrm{er}}$ : "when the cultivated soil joins the rocky flat, exactly at the spot where the road turns off to the right to go to the tombs of the kings " (St. John, 1845 : 364365). Aux tombeaux creusés dans la montagne étaient par ailleurs assignées diverses fonctions au moment des crues du Nil, qui, à l'époque, recouvraient l'étroite bande de terres agricoles jusqu'aux pieds des colosses de Memnon : habitations provisoires, réserves de denrées ou encore refuge pour les hommes recherchés par la police ou l'armée lors des campagnes musclées de conscription ou de rébellion paysanne. Les sites archéologiques sont aussi une source de revenus pour certains. La cachette des momies de Deir al-Bahari, découverte en 1881 par Maspero et son équipe, aurait fait vivre pendant une décennie les tribus de la montagne. L'histoire nourrit les imaginaires et fonde la mauvaise réputation des hommes de Louxor depuis plus d'un siècle. Les guides touristiques en ont fait l'anecdote locale, certains héritiers leur fonds marketing touristique, et le cinéaste égyptien Shady Abd al-Salâm en a tiré un très beau film à la fin des années $1960^{11}$.

\section{Carte 1}

\section{Rive est de Louxor (Égypte) en 1891}

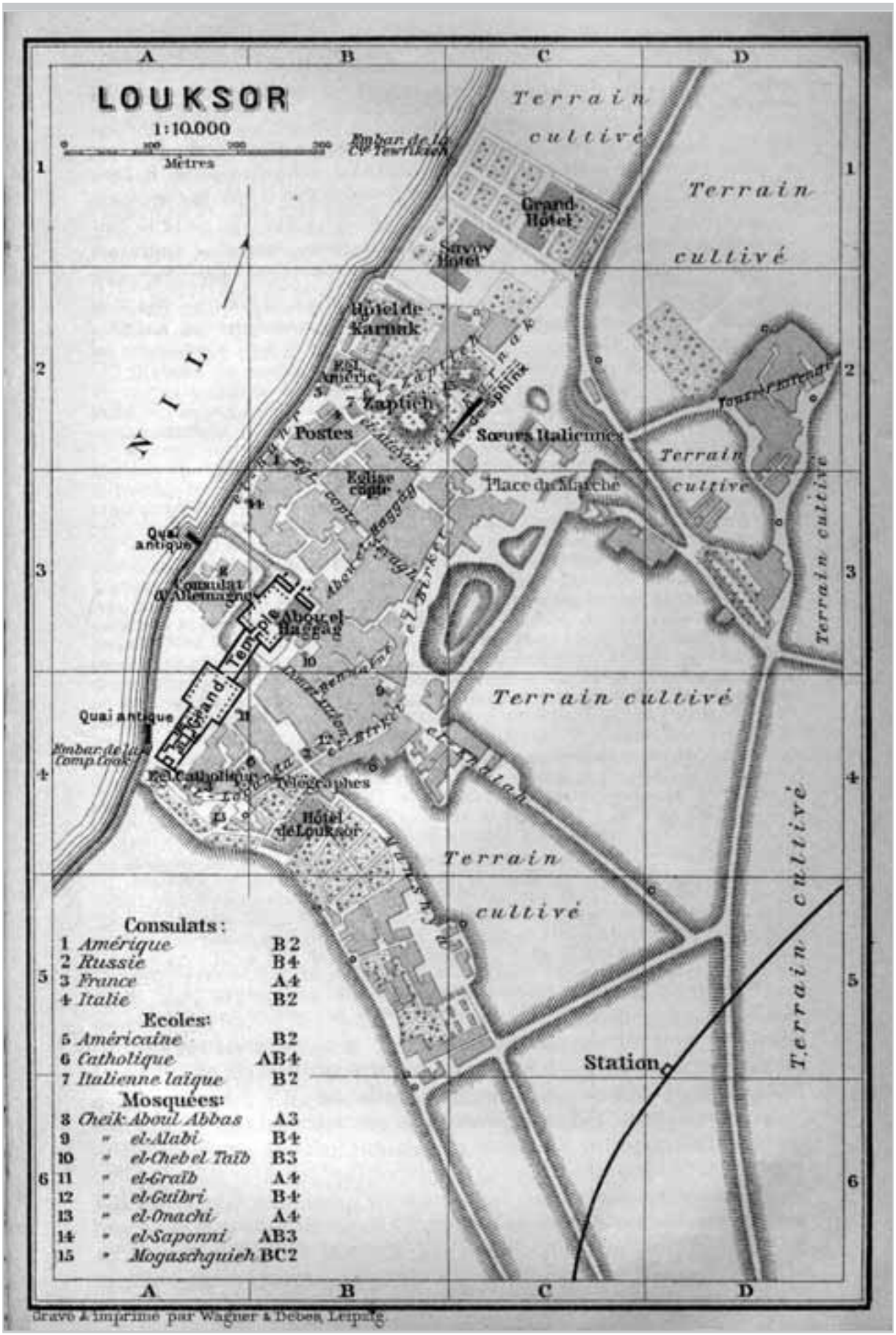

Source : Guide Baedecker, 3 é édition française,1903, collection personnelle de l'auteure.

Les dégradations et les menaces faites au temple de Louxor par la croissance de la population et les activités touristiques ${ }^{12}$ encouragent Maspero, alors directeur du service des antiquités égyptien, à lancer un vaste programme de déblaiement et de res- tauration à partir de 1881. II s'agit de déplacer le village ainsi que les résidences consulaires, installées dans l'enceinte du site. Dès lors, la population du «village arabe» de Louxor s'installe progressivement hors de l'enceinte du temple, et bien 
au-delà. Le programme se fait notamment avec le soutien financier de l'agence Thomas Cook.

\section{En 1882, Maspero prend contact avec la maison Cook et Cie, principal entrepre- neur de voyages sur le Nil, et lui deman- de son avis sur l'aide que pourraient ap- porter les touristes. Cook devient agent de liaison entres les touristes et le service des Antiquités : il transmet les doléances et si- gnale les déprédations, qu'elles soient le fait de visiteurs indélicats, d'agents du ser- vice, ou d'habitants inconscients (David, 1999: 119).}

Plus qu'agents de liaison, Cook et sa famille sont directement impliqués dans les fouilles et financent personnellement une partie des travaux de déblaiement du temple ${ }^{13}$.

Ainsi, à partir des années 1880, la convergence des intérêts touristiques et scientifiques entraîne une transformation radicale de l'organisation des lieux, à coup de pelles et de burins des égyptologues et sous la pression des touristes occidentaux férus de monuments pharaoniques et d'aventures orientales. Louxor connaît sur la période 1882-1897 une croissance remarquable pour une bourgade rurale de Haute-Égypte, passant respectivement de moins de 6000 à plus de 10000 habitants, soit une croissance annuelle de 4,1\%, à l'instar du village de Gurna, sur la rive ouest, en plein cœur de la nécropole thébaine (soit 3,6 \% de croissance annuelle sur la même période). En 1907, Louxor et les deux villages de Karnak et de Gurna comptent ensemble près de 30000 habitants $^{14}$. Ce sont là trois hauts lieux majeurs de concentration de monuments antiques, d'attraction et de sédentarisation de nouvelles populations (la région est chargée d'histoire de tribus plus ou moins nomades), tant pour fournir en maind'œuvre les missions archéologiques que pour occuper une activité directement ou indirectement liée au tourisme.

De 1880 à 1910, Louxor connaît du même coup un développement particulièrement important de ses infrastructures touristiques, passant de quelques dizaines de chambres d'hôtel à près de $500^{15}$. L'agence Thomas Cook inaugure en 1877 le premier établissement, l'hôtel de Louxor, et conserve le monopole sur l'accueil hôtelier pendant plus d'une décennie. Jusqu'alors, les voyageurs pouvaient être logés dans les maisons des représentations consulaires ou par les missions archéologiques. En 1890, l'hôtel de Karnak (toujours de l'agence Thomas Cook) vient s'ajouter au premier, pendant qu'Andrawos Bacha, notable de Louxor et grand propriétaire terrien, offre un terrain à une agence concurrente pour y ouvrir l'hôtel Tawfiqiya, situé sur les berges du Nil. Louxor possède alors un embarcadère, géré par Thomas Cook, pour l'accueil des dahabiya et des bateaux à vapeur, une poste, un télégraphe. L'hôpital « indigène ", qui accueille des patients venant de toute l'Égypte, est inauguré en 1891 sur l'initiative de Thomas Cook et financé, dans la tradition philanthropique britannique, par des fonds récoltés auprès des touristes. Enfin, une ligne de chemin de fer relie Le Caire à Assouan à partir de 1897. "Les wagons sont pareils à ceux de France et d'Italie. Un Européen ne trouvera qu'en 1 ère classe le confort auquel il est habitué » (Guide Baedecker, 1903). En saison, un train de luxe est en fonction, trois fois par semaine; deux express partent quotidiennement pour Louxor. Jusqu'à la construction au nord du pont de Naga' Hamâdî en 1897, la ligne de chemin de fer ne passait pas au-delà d'Assiout. II fallait alors embarquer sur un bateau à vapeur pour rejoindre Louxor (Cinq semaines en Égypte, 1903). Depuis, «la station de Louxor est admirablement aménagée pour la réception des touristes " (Comment visiter l'Égypte, 1911-1912).

Au tournant du siècle, l'offre d'accueil en hôtel se diversifie: en 1903, le touriste peut résider dans une "pension de famille " au prix modeste et « très convena- ble » (Guide Baedecker : 1903), ou encore au tout nouveau Savoy Hotel. En 1907, le Winter Palace de Thomas Cook est inauguré et prêt à accueillir la bonne société cosmopolite d'Égypte et du monde: "Ses aménagements sont tout ce qu'il y a de plus moderne et de plus luxueux, lumière électrique et ascenseur " (Comment visiter l'Égypte, 1911-1912). La petite ville de Louxor est sans conteste "moderne", adaptée aux standards d'une clientèle internationale de plus en plus nombreuse et de la bonne société égyptienne.

La bourgade de Louxor possède au début du siècle les attributs d'une urbanité qui s'apparentent à ceux d'une capitale, toutes proportions gardées. Y convergent toutes sortes de commerces:

Dans l'alignement pompeux du Winter Palace, des boutiques se succèdent: on y vend tout ce dont s'affublent les touristes : éventails, chasse-mouches, casques et lunettes bleues. En plus la bimbeloterie du Soudan: vieux couteaux de nègre, peaux de panthère et cornes de gazelles. Même les Indiens sont venus en foule à cette foire improvisée, apporter les étoffes du Radjapoute ou du Cachemire (Loti, 1909: 213).

S'y pratiquent toutes sortes d'activités qui paraissent pour le coup incongrues pour le lieu et en révèlent la forte urbanité : chapeliers, prostituées, tailleurs à l'européenne, photographe ou encore un réparateur de piano.

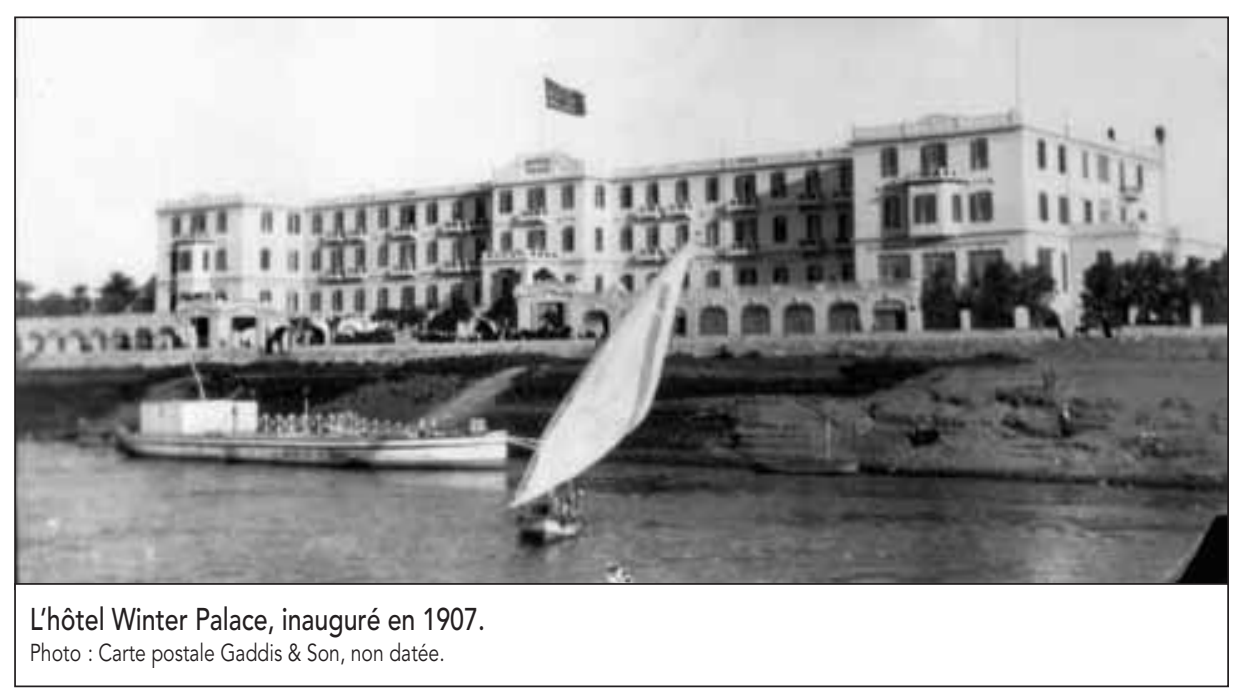


À mesure que le nombre des touristes s'accroît, l'offre d'accueil se diversifie. Au début des années 1920, on compte une demi-douzaine de petits hôtels récents, situés notamment dans le quartier de la gare. La découverte du tombeau de Toutankhamon en novembre 1922 relance l'égyptomanie occidentale et le rêve du voyage en Orient, entraînant une augmentation notable de visiteurs étrangers en Égypte $^{16}$. Elle devient aussi affaire politique, plaçant Louxor au cœur des enjeux nationalistes égyptiens: l'Égypte vient d'accéder à l'indépendance, aussi formelle soit-elle, et les gouvernements qui se succèdent ont alors un souci tout particulier à l'égard de Louxor, haut lieu de l'histoire nationale, du tourisme international et territoire hautement disputé. Le gouvernement de Sa'ad Zaghlûl, grande figure du mouvement indépendantiste égyptien, décide en février 1924 de "nationaliser ", en quelque sorte, le tombeau de Toutankhamon. L'ouverture du tombeau au public se transforme alors en manifestation politique, en présence de quelque 170 personnalités égyptiennes qui ont fait le voyage du Caire pour l'occasion. Lord Allenby, haut commissaire britannique, est accueilli par les cris de la foule de Louxor, appelant au retrait de la Grande-Bretagne de l'Égypte (Reid, 1985 et 1997).

Égyptomania occidentale et intérêt nationaliste égyptien relancent dans les années 1920 les plans d'aménagement et d'embellissement de l'ancienne capitale thébaine. La corniche est dotée d'éclairage et de nouveaux débarcadères; les bâtiments de style néo-pharaoniques soulignent les percées de la ville touristique et moderne (la gare, la station d'électricité); les maisons bourgeoises révèlent l'enrichissement de la population et le dynamisme des activités de la ville. Quant à l'agence Thomas Cook, son monopole sur Louxor s'effrite à mesure que les touristes deviennent plus nombreux et la concurrence internationale plus ardue. Les stratégies d'expansion de la compagnie portent vers d'autres horizons ${ }^{17}$, pendant que la tutelle britannique s'affaiblit inexorablement face aux mouvements nationalistes émergents et aux velléités de contrôle des autorités égyptiennes sur ses territoires les plus emblématiques de l'occupation étrangère, à l'instar de Louxor et de ses sites antiques.

\section{En guise d'épilogue : Louxor mondialisé 18}

L'Égypte, avec ses 8,5 millions de touristes en 2005, représente aujourd'hui une part résiduelle du flux touristique mondial et un marché plus que marginal pour la compagnie Thomas Cook. À l'occasion, le rôle de l'agence dans l'histoire moderne de l'ancienne capitale thébaine et dans celle du tourisme en Égypte est rappelé à ses représentants locaux, sans pour autant susciter de grands émois ou même encore d'intérêt marketing. Force est d'admettre que les choix de développement touristique retenus dans la région de Louxor ainsi que les stratégies de développement mises en œuvre dans la région par les autorités égyptiennes, les agences internationales de développement et les professionnels du secteur touristique ne mobilisent guère, à l'heure du tourisme de masse, les nostalgiques du voyage en Orient ${ }^{19}$. Loti parlait d'un Louxor modernisé au début du siècle dernier; une modernisation incarnée par le vapeur, le traveler check et le voyage en groupe. De nos jours, pourrait-on parler d'un Louxor mondialisé ; une internationalisation qui s'exprime par un plan d'aménagement fondé sur trois principes directeurs de spécialisation des territoires:

Principe d'open-air museum: II s'agit en premier objectif de faire de Louxor une ville-musée, exclusivement dédiée aux visiteurs internationaux et aux monuments antiques.

Principe d'enclave touristique : Une politique de l'offre, qui consiste à augmenter la capacité d'accueil touristique (deux nouvelles villes touristiques sont en projet), permet d'aligner les conditions d'accueil sur les standards exigés par une clientèle touristique essentiellement occidentale (critères de confort et d'esthétique).

Principe de déplacement des populations: L'histoire de Louxor nous montre combien monuments et populations sont étroitement liés. II s'agit aujourd'hui de vider les sites antiques des populations qui les occupent et de reloger ces derniers dans des villes nouvelles, situées dans le désert, loin des centres d'activités touristiques qui constituent pourtant les seules sources de revenus dans la région. La population concernée est estimée à plusieurs milliers : une grande partie du villa- ge de Gurna sur la rive ouest, soit environ 2000 foyers, et les résidants de la ville de Louxor, qui habitent entre le temple de Louxor et celui de Karnak, afin de mettre à jour la totalité de l'allée des sphinx qui relie les deux temples.

De telles mesures semblent nécessaires, pour le moins aux yeux des autorités égyptiennes et des professionnels du tourisme, face aux contraintes d'espace, à l'augmentation de la population, sa promiscuité avec les touristes et les monuments, mais aussi face aux dégradations faites aux sites archéologiques, inscrits en 1979 par I'Unesco (Organisation des Nations unies pour l'éducation, la science et la culture) sur la liste du Patrimoine mondial comme biens culturels. De telles mesures d'aménagement permettent aussi et surtout de mettre en place les conditions propices à une circulation touristique sécurisée et balisée par les professionnels du tourisme, égyptiens et internationaux. Le chantier mis en œuvre aujourd'hui devra à terme transformer radicalement la physionomie de l'ancienne capitale thébaine, au nom de la protection du monument, certes, mais surtout au regard d'un donné crucial de développement économique: le tourisme, première rente du pays et source première d'emplois et de revenus dans la région. À ce titre, Louxor demeure un territoire hautement stratégique et disputé, où se jouent des enjeux qui dépassent le cercle local. Son développement dans les années à venir devrait être instructif des choix politiques et des contraintes imposées, là où les intérêts ne sont pas toujours forcément convergents, entre protection des monuments, développement touristique et populations.

Sandrine Gamblin est politologue et arabisante, associée au Centre d'études et de documentation économiques et juridiques.

\section{Notes}

1 Les photos $n^{\circ} 1$ et $n^{\circ} 3$ sont tirées des archives familiales d'Ihab Gaddis dont le grandpère photographe, A. Gaddis, possédait un studio à Louxor au début du siècle, situé à partir de 1907 et jusqu'à nos jours au pied du Winter Palace. Le fonds Gaddis, riche de près de 3000 clichés, couvre plusieurs décennies d'histoire de Louxor, témoignant de l'activité archéologique (photos de monuments) et touristique, mais aussi d'événements politiques (visites officielles) et de faits de la vie quotidienne (portraits, scènes de rue). Nous 
remercions chaleureusement M. Ihab Gaddis de nous avoir accordé l'autorisation de publier ces deux clichés.

2 Pour être plus précis, deux bateaux de croisière sont au départ affrétés par l'agence Cook. Mais seul un bateau est autorisé à naviguer sur le Nil, le second restera bloqué à Alexandrie, pour rejoindre la Palestine ultérieurement.

3 Miss Riggs, Diary " Grand Tour to the Nile and Palestine », 1869 (consulté aux archives de Thomas (ook).

4 Le collectif est publié dans le London Times du 9 juin 1874 (Brendon, 1991).

5 Plus de $30 \%$ de la population urbaine; une proportion qui se maintient jusqu'à nos jours (source : Recensement national de la population, 1896-1986). Sur les relations entre coptes et musulmans à Louxor, on lira le roman de Baha Taher (1996), Tante Safeya et le monastère, traduit en français.

6 L'une des villas accueille le quartier général du Parti national démocrate (parti au pouvoir) ; la seconde est encore habitée par les descendantes des Andrawos, que l'on appelle communément à Louxor les «trois Françaises ". Un roman est consacré à la saga familiale (Asaad, 1992).

7 Journal publié par la compagnie Thomas Cook \& Son, 1876

8 Lettre de Maspero à Scott Moncrieff (soussecrétaire d'État au ministère des Travaux publics), publiée dans le Journal des Débats du 26 février 1884 (David, 1999: 123). Quant à la présence permanente d'étrangers à Louxor, elle s'établit autour de 200 individus en 1907 et atteint son maximum en 1927, avec près de 700 étrangers recensés (recensements nationaux de la population).

9 Lettre de Lefébure à Maspero, 30 décembre 1881 (David, 1999 : 87).

10 " [...] c'est une grande maison en partie construite avec des matériaux antiques audessus du sanctuaire d'Alexandre; des fûts brisés, des piliers sciés en deux, des pierres chargées d'inscriptions soutiennent les murs bâtis en brique et crépis à la chaux ; c'est la Maison de France. Les officiers de marine qui firent partie de l'expédition du Louksor [1826] y logèrent, et depuis elle appartient au gouvernement français, auquel Méhémet-Ali l'a verbalement donnée. Un magnifique jardin verdoie derrière; ce sont nos marins qui l'ont planté » (Du Camp, 1857 : 215).

11 Film La Momie, sorti en 1969 sur les écrans.

12 En 1879, les autorités locales avaient eu un temps l'idée de vendre les terrains du temple à un " promoteur » touristique, lequel envisageait de détruire une dizaine de colonnes de la cour principale afin d'y construire un hôtel. Lettre de Lefébure à Maspero, 30 décembre 1881 (David, 1999: 87)
13 Lettre à Louise, 28 janvier 1886. La femme de Maspero y fait état d'une visite à Louxor de la famille Cook (David, 1999, p. 120).

14 Les données démographiques sont tirées des recensements nationaux de population.

15 Les chiffres correspondent à l'information donnée par les principaux guides touristiques de l'époque.

16 On enregistre pour la saison 1925-1926 plus de 15000 touristes en Égypte (chiffres disponibles) (Cattaui, 1926).

17 Thomas Cook et son fils John meurent à la fin des années 1890, laissant la compagnie aux trois fils de ce dernier. L'agence est finalement vendue en 1928 à la société belge des Wagons-lits et des Grands Express Européens (Brendon, 1991), marquant ainsi la fin du monopole de Thomas Cook sur le marché touristique international.

18 Pour un développement sur Louxor contemporain, voir Gamblin (2004).

19 II existe cependant une demande, constitutive d'une niche très lucrative, mais limitée sur le marché touristique. Ainsi, des dahabiyas sont à nouveau en circulation sur le Nil entre Louxor et Assouan, proposant une croisière « à l'ancienne " avec décor 1900 réajusté et circuits hors des sentiers battus.

\section{Bibliographie}

A Handbook For Travellers in Lower and Upper Egypt (1880), London, John Murray, tome II.

Asaad, Fawzia (1992), La Grande maison de Louxor, Paris, l'Harmattan, Lettres arabes (roman).

Baedeker, Karl (1898, première édition française), Égypte, manuel du voyageur, Leipzig, Édition Karl Baedeker.

Berchet, Jean-Claude (1985), Le voyage en Orient, anthologie des voyageurs français dans le Levant au XIXe siècle, Paris, R. Laffont.

Brendon, Piers (1991), Thomas Cook, 150 Years of Popular Tourism, London, Secker and Warburg.

Cattaui Pacha, Joseph (1926), "Le tourisme ", dans L'Égypte. Aperçu historique et géographique, gouvernement et institutions, vie économique et sociale, Le Caire, Institut français d'archéologie orientale, p. 409-452.

Chih, Rachida (1993), "Abu-I-Hajjâj al-Uqsûrî, saint patron de Louqsor. Égypte/Monde Arabe, Le Caire, Centre d'études et de documentation économiques et juridiques, $n^{\circ} 14$, p. 67-78.

Cinq semaines en Égypte Alexandrie, Le Caire, Thèbes, Assouan. Notes de voyage (1903), Paris, Imprimerie Fortin et Cie, p. 61.

Comment visiter l'Égypte, (1911-1912), Paris, Hachette.

Daressy, Georges (1893), Notices explicatives des ruines du temple de Louxor, Le Caire, Imprimerie nationale, Service des antiquités de l'Égypte.
David, Elisabeth (1999), Gaston Maspero 18461916, Le gentleman égyptologue, Paris, Pygmalion.

Du Camp, Maxime (1857), Le Nil. Égypte et Nubie, Paris, Hachette.

Dyab, al-Sayd (1994), Le Tourisme en Égypte durant le $X I X^{e}$ siècle (en arabe).

Gamblin, Sandrine (2004), "A Tale of Two Cities: Tourism and Heritage Management in Luxor ", dans Nicholas Hopkins et Reem Saad, Social and Economic Process in Upper Egypt, Le Caire, The American University in Cairo Press.

Humbert, Jean Marcel, Michael Panfazzi, et Christiane Ziegler (1994), Égyptomania. L'Égypte dans l'art occidental, Paris, Réunion des musées nationaux.

Legrain, Georges (1914), Louqsor sans les Pharaons. Bruxelles/Paris, Vromant.

Legrain, Georges (1916-1917), "Rapport sur les nouveaux travaux exécutés à Lougsor à l'ouest du temple d'Amon (octobre 1916 -mars 1917)". Annales du service des antiquités de l'Égypte, tome XVII, p. 55-58.

Loti, Pierre (1909), La Mort de Philae, Paris, Calman Lévy.

Maspero, Gaston (1901, 2000), Ruines et paysages d'Égypte, Paris, Payot.

Nelson, Nina (1997), The History of Mena House Hotel. Cairo, Egypt, Cairo, The Palm Press.

Rauch, André (2000), «L'Orient dans l'essor du tourisme au XIX ${ }^{e}$ siècle ", Relations internationales, $n^{\circ} 102$, p. 161-172.

Reid, Donald (1985), «Indigenous Egyptology. The Decolonization of a Profession?", Journal of the American Oriental Society, $n^{\circ} 105$, p. 233-246.

Reid, Donald (1997), "Nationalizing the Pharaonic Past: Egypt, 1922-1952 ", dans James Jankowski et Israel Gershoni, Rethinking Nationalism in the Arab Middle East, New York, Columbia University Press, p. 127-149.

Reid, Donald (2002), Whose Pharaohs? Archaeology, Museums and Egyptian National Identity from Napoleon to World War I, Cairo, The American University in Cairo Press.

St. John, James (1845), Egypt and Nubia. Éditeur non spécifié

Stevens, G.W (1898), Egypt in 1898. London, William Blackwood and Sons.

Taher, Baha (1996), Tante Safeya et le monastère, Paris, Autrement (roman).

Urbain, Jean Didier (1991), L'ldiot du voyage. Histoires de touristes, Paris, Albin Michel.

Veblen, Thorsten (1899/1970). Théorie de la classe des loisirs. Paris, Tel / Gallimard.

Volkoff, Oleg (1967), Comment on visitait la vallée du Nil : Les guides de l'Égypte. 